\title{
Исследование электростатической системы поверхности кристаллов AuNi/GaN диодов Шоттки методом зонда Кельвина атомно-силовой микроскопии
}

\author{
(C) Н.A. Торхов ${ }^{1,2,3}$, В.А. Новиков ${ }^{2}$ \\ ${ }^{1} \mathrm{AO}$ „НИИПП“, \\ 634034 Томск, Россия \\ ${ }^{2}$ Национальный исследовательский Томский государственный университет, \\ 634050 Томск, Россия \\ ${ }^{3}$ Томский университет автоматизированных систем управления (ТУСУР), \\ 634050 Томск, Россия \\ E-mail: trkf@mail.ru
}

Поступила в Редакцию 3 июля 2019 г.

В окончательной редакции 20 сентября 2019 г.

Принята к публикации 21 октября 2019 г.

\begin{abstract}
Атомно-силовые микроскопические исследования электростатической системы поверхности кристаллов плоских $\mathrm{AuNi} / n-n^{+}-\mathrm{GaN}$ диодов Шоттки показали, что значение работы выхода электронов с поверхности металлических контактов Шоттки зависит от их линейного размера - диаметра $D$. При $D>120$ мкм значение работы выхода центральной области контактов приближается к значению работы выхода сплошной металлической пленки золота $e \varphi_{\text {Аu }} \approx 5.40$ эВ. Уменьшение диаметра приводит к уменьшению работы выхода до 5.34 эВ для $D=120$ мкм, 5.21 эВ для $D=40$ мкм, 5.18 эВ для $D=10$ мкм и 5.14 эВ для $D=5$ мкм. Наблюдаемое уменьшение значений работы выхода с уменьшением диаметра связано с увеличивающимся влиянием встроенного электростатического поля периферии $\mathbf{E}_{l}$, которое определяется площадью и периметром контактов Шоттки. Принципиальные отличия термодинамических и электростатических систем омических TiAlNiAu $/ n^{+}-\mathrm{GaN}$-контактов (в отличие от аналогичных систем $\mathrm{AuNi} / n$-GaN-контактов Шоттки) указывают на отсутствие в них барьера Шоттки и преобладающую роль термоэмиссионного механизма переноса подвижных носителей электрических зарядов.
\end{abstract}

Ключевые слова: нитрид галлия, барьер Шоттки, омический контакт, электростатическое поле периферии, размерный эффект, метод зонда Кельвина АСМ.

DOI: 10.21883/FTP.2020.03.49031.9203

\section{1. Введение}

Барьер Шоттки (БШ) как физический объект представляет собой сформированный на интерфейсе металлполупроводник (M-П) управляемый внешним электрическим полем потенциальный барьер, уникальные свойства которого обусловливают его широкое применение в электронике. Физические и приборные модели контактов М-П с БШ (контакт Шоттки) достаточно подробно описаны в современной научной литературе и в настоящее время продолжают уточняться и развиваться [1-5].

Согласно [1-5], возможностей диффузионной и термоэмиссионной теорий (основанных на диффузионном механизме переноса носителей электрических зарядов с учетом процессов рассеяния) достаточно для описания параметров большинства низкочастотных (с диаметром $D$ контакта, превышающим десятки и сотни микрометров) контактов Шоттки с толстыми ( $\geq 0.5$ мкм) базами. При этом для расчета приборных характеристик используется всего два параметра барьера Шоттки: его высота $\varphi_{b}$ и ширина области пространственного заряда (ОПЗ) $W[1]$.

При переходе к описанию высокочастотных характеристик контактов Шоттки (диаметрами от едини- цы микрон и менее) с тонкими (<0.5 мкм) базами уже необходимо применение термополевой, а лучше баллистической (тонкую базу электроны проходят без рассеяния) теорий переноса баллистических носителей электрических зарядов в контактах Шоттки с учетом полной формы потенциального барьера [6]. Согласно [7], для описания токопрохождения в таких контактах абсолютно недостаточно параметров $\varphi_{b}$ и $W$, а необходимо учитывать взаимодействие (рассеяние) электронов с полной формой потенциального барьера, включая и область, в которой электроны движутся над барьером.

В настоящий период времени реализация таких контактов традиционно осуществляется путем химического или физического нанесения барьерной металлизации на подготовленную поверхность различных полупроводниковых (узкозонных [8], широкозонных [2-5,9], прямозонных $[10,11]$ и непрямозонных [12]) материалов, включая и органические полупроводники [13].

Умение эффективно управлять свойствами таких контактов открывает широкие возможности их практического использования в приборах в различных областях современной науки и техники для измерения физикохимических и электрофизических параметров различных 
объектов, контроля и управления быстропротекающими процессами, ключах, детектирования, обнаружения элементарных частиц и квантов излучения, сверхскоростного преобразования и передачи сигналов в высокочастотных оптоволоконных и квантовых линиях связи, спинтронике и квантовых компьютерах, радиовидении, ближней и космической локации, генерации, распространения и поглощения сверхвысокочастотного (СВЧ), крайне высокочастотного (КВЧ), терагерцового (ТГц) радио- (далее высокочастотное радиоизлучение) и оптического излучений, в изучении взаимодействия этих излучений с биологическими объектами на генном и молекулярном уровнях, в радиофотонике и энергоэффективных устройствах преобразования и передачи лучистой энергии и т. п.

Интенсивное развитие материаловедения и 0D, 1D и 2D - нанотехнологий (квантовые точки и провода, двумерный электронный газ и др.) создало технологическую базу для качественно нового этапа в изготовлении и изучении свойств контактов Шоттки субмикронных и наноразмеров (например, [14]).

Несмотря на большой объем имеющихся результатов и продолжающихся исследований, многие свойства контактов М-П с БШ, касаемые их электрофизической природы и механизмов токопрохождения, все еще остаются недостаточно хорошо изученными. Это накладывает серьезные ограничения на эффективность их практического использования для достижения предельных технико-эксплуатационных характеристик разрабатываемых устройств.

Так, остаются открытыми вопросы о физической природе эффекта „резонанса горячих электронов“, приводящего к увеличению в рамках теории Фаулера [15] внешней квантовой эффективности фотодиодов с барьером Шоттки [16], нетривиальном влиянии формы контактов Шоттки на их статические и высокочастотные характеристики, вольтовую $\beta_{U}$ и токовую $\beta_{I}$ фоточувствительности [17]. В частности, в рамках известных представлений о пропускной способности барьера Шоттки невозможно объяснить тот факт, что в микронных контактах Шоттки к широкозонным полупроводникам амплитуда фотоэлектродвижущей силы (фотоэдс) $U_{\mathrm{Ph}}$, как и сила фототока $I_{\mathrm{Ph}}$ нетривиальным образом зависит от геометрической формы контактов, а обратные токи насыщения $I_{S, r}$ значительно (на несколько порядков) могут превышать прямые токи насыщения $I_{S, f}[17]$.

Напомним, что контакт М-П с БШ бесконечной площади $(S \rightarrow \infty)$ лишен аддитивных геометрических свойств и, как следствие, не имеет аддитивных приборных характеристик, его емкость бесконечна, а сопротивление барьера стремится к нулю и в пределе при $S \rightarrow \infty$ не зависит от смещения, т.е. не имеет нелинейных выпрямляющих (диодных) характеристик [17]. Значительная роль периферии проявляется в том, что контакт Шоттки приобретает нелинейные аддитивные электрические характеристики только после формирования у него границы (периферии) и появления аддитивных геометрических свойств.

Таким образом, уменьшение линейных размеров контактов Шоттки приводит к возрастающему влиянию периферийной области на их электростатическую систему, форму потенциального барьера Шоттки и, как следствие, на их статические и высокочастотные приборные характеристики. Такое влияние обеспечивается электрическими полями периферии, экранирующими термодинамическую систему контакта Шоттки от окружающей среды $[4,17]$.

Возможности современных методов атомно-силовой микроскопии (ACM) позволили прецизионно исследовать систему электростатических полей периферии плоских контактов М-П с БШ на арсениде галлия и обнаружить зависимость значений работы выхода электронов $A_{e}=e \varphi_{\mathrm{Au}}$ (далее - работа выхода) с поверхности металлических контактов от их линейных размеров (диаметров $D$ ) - латеральный размерный эффект [18-21]. Было показано, что зависимость $A_{e}=A_{e}(D)$ обусловлена влиянием встроенного электростатического поля периферии $\mathbf{E}_{l}=\mathbf{E}_{l}(D)$, модуль которого $\left|\mathbf{E}_{l}\right|$ изменяется при изменении параметра $\xi=P / S$, где $P$ - периметр контакта. Было обнаружено, что для арсенида галлия $n$-типа проводимости (n-GaAs) уменьшение диаметра $D<500$ мкм приводит к значительному уменьшению работы выхода $A_{e}$, а для арсенида галлия $p$-типа проводимости наоборот - к ее увеличению [22,23]. Подобные эффекты были замечены также для плоских $P t$ - [24] и $R h P t$-контактов Шоттки [25] на $n$-GaAs, а также для контактов Шоттки на фосфиде индия [26].

Несмотря на вышеизложенное, исследования электростатической системы плоских контактов Шоттки на широкозонных полупроводниках крайне ограничены. В связи с этим представляет научно-практический интерес проведение исследований электростатической системы контактов $\mathrm{M}-П$ с БШ на нитриде галлия $n-\mathrm{GaN}$ электронного типа проводимости в связи с его активным использованием в полупроводниковых приборах разного назначения.

\section{2. Методическая часть}

Для изготовления кристаллов планарных диодов Шоттки использовалась выращенная на подложке сапфира эпитаксиальная $n-n^{+}-\mathrm{GaN}(0001)$-структура. Толщина эпитаксиального слоя $n$-GaN с концентрацией $N_{D} \approx 5 \cdot 10^{16} \mathrm{~cm}^{-3}$ составляла $180 \mathrm{HM}$, слоя $n^{+}-\mathrm{GaN}$ с концентрацией $2 \cdot 10^{18} \mathrm{~cm}^{-3}-5$ мкм. Со стороны эпитаксиального слоя $n-\mathrm{GaN}$ методом плазменного травления изготавливались мезы высотой 200 нм. Затем вокруг мез на поверхности высоколегированного слоя $n^{+}$-GaN методом взрывной литографии формировались омические TiAlNiAu-контакты с последующим быстрым 
термическим отжигом в атмосфере азота при температуре $780^{\circ} \mathrm{C}$. После этого на поверхности эпитаксиального $n$-слоя методом взрывной литографии формировались AuNi-контакты Шоттки диаметрами $D_{1}=5$, $D_{2}=10, D_{3}=40$ или $D_{4}=120$ мкм. Толщина Ni составляла $50 \mathrm{Hм}, \mathrm{Au}-200 \mathrm{нм}$.

Контроль геометрических размеров изготовленных контактов (рис. 1, $a$ и $b$ ) и электростатического потенциала поверхности $\varphi_{s}(m)$ (работы выхода электронов $\left.A_{e}=e \varphi_{s(m)}\right)$ (1) (рис. $1, c$ и $d$ ) проводился на атомно-силовом микроскопе „Solver-HV“ (производства NT MDT) в двухпроходном режиме метода зонда Кельвина. Влияние элементов конструкции кантилевера на результаты измерений - так называемое приборное влияние - не превышало $2.5 \%$ на расстоянии 5 мкм от контакта и $10 \%$ в непосредственной близости от него:

$$
A_{e}=e \varphi_{s(m)}=e \varphi_{p}-e \Delta \varphi_{s(m)}
$$

Здесь $e=1$ - заряд электрона в системе СГС, $e \varphi_{p}\left(\mathrm{~W}_{2} \mathrm{C}\right)=4.92$ эВ - работа выхода иглы кантилевеpa $\left(\mathrm{HA}-\mathrm{FM} / \mathrm{W}_{2} \mathrm{C}\right)$ с покрытием из карбида вольфрама $\mathrm{W}_{2} \mathrm{C}, \varphi_{s(m)}$ - электростатический потенциал (далее - потенциал) исследуемой поверхности полупроводника $(s)$, или металлического $(m)$ контакта, $\Delta \varphi_{s(m)}-$ измеряемая АСМ-методом зонда Кельвина контактная разность потенциалов (КРП).

Измерения статических вольт-амперных характеристик (BAX) проводились на зондовой станции „Cascade-M150“ с использованием измерителя электрических характеристик полупроводниковых приборов B1500А производства Keysight Technologies (рис. 1,e).

\section{3. Результаты экспериментов}

Изготовленные $\mathrm{NiAu} / n-\mathrm{GaN}$-контакты Шоттки имели классические ВАХ с показателем идеальности $n=1.06-1.1$ и высотой барьера $e \varphi_{b}=0.93-0.98$ эВ (рис. 1,e). Это позволило интерпретировать полученные результаты с использованием известных физических представлений о выпрямляющих контактах металлполупроводник с барьером Шоттки.

Согласно рис. 1, латеральное распределение электростатического потенциала исследуемых контактов Шоттки на $n$-GaN аналогично латеральному распределению электростатического потенциала плоских $\mathrm{Me} / n$-GaAsконтактов Шоттки на $n$-GaAs.

В частности, из рис. 1, $a$ следует, что работа выхода электронов из $\mathrm{NiAu-контактов} \mathrm{Шоттки} \mathrm{к} \mathrm{нитриду}$ галлия, как и в случае с контактами Шоттки на арсениде галлия, значительно превышает работу выхода электронов $A_{e}$ с поверхности сплошной пленки NiAu. А именно барьерный $\mathrm{NiAu-контакт,} \mathrm{как} \mathrm{и} \mathrm{металлические}$ контакты Шоттки на GaAs, не находится в термодинамическом равновесии со всем объемом эпитаксиального слоя $n$-GaN. При этом работа выхода электронов с поверхности $\mathrm{NiAu} / n-\mathrm{GaN}$-контактов так же, как и в случае с плоскими контактами $\mathrm{Me} / n-\mathrm{GaAs}$, имеет зависимость от диаметра $D$ (рис. 2). Так, для $\mathrm{AuNi} / n-\mathrm{GaN}$ контактов Шоттки диаметрами $D \gg 120$ мкм значение работы выхода приближается к значению работы выхода сплошной золотой пленки $е \varphi_{\text {Au }}=e \varphi_{\text {Аu }}($ film $)=5.40$ эВ (рис. $2, d$ ). Согласно полученным результатам, уменьшение диаметра приводит к уменьшению работы выхода $\mathrm{AuNi} \mathrm{NiAu} / n-\mathrm{GaN}$-контактов Шоттки, которая для $D=5$ мкм составляет $e \varphi_{(m)}(\mathrm{Au})=5.14$ эВ. Вне периферии контакта наблюдается переходная область плавно изменяющегося потенциала — „ореол“ протяженностью $l^{*}$, характерный и для плоских контактов Шоттки на арсениде галлия, а также переходная область внутри периферии - „ореол“ протяженностью $l_{C}^{*}$ (рис. 2, d).

Согласно [24-31], результирующий вектор электростатического поля периферии $\mathbf{E}_{l}$ образован суперпозицией векторов постоянного электростатического поля $\mathbf{E}_{\text {grad }}$, образованного разностью поверхностных потенциалов металлического контакта и свободной полупроводниковой поверхностью, и вектора нескомпенсированного электростатического поля $\mathbf{E}^{*}$, выходящего через боковую поверхность ОПЗ:

$$
\mathbf{E}_{l}=\mathbf{E}_{\text {grad }}+\mathbf{E}^{*}, \text { и для модулей }\left|\mathbf{E}_{l}\right|=\left|\mathbf{E}_{\text {grad }}\right|-\left|\mathbf{E}^{*}\right| .
$$

Модуль $\left|\mathbf{E}^{*}\right|=E^{*}$ зависит от формы (площади $S$ и периметра $P$ ) контакта. Согласно [30,31], встроенное электростатическое поле $\mathbf{E}_{l}$ формирует в области контакта встроенный электростатический потенциал $\varphi^{*}$ (рис. $2, c, d)$, который, как было показано в [17], оказывает значительное влияние на работу выхода электронов из металлических контактов Шоттки.

Согласно рис. $1, d$, максимум электростатического поля $E^{*}$ приходится на периферию выпрямляющего $\mathrm{AuNi- \kappa онтакта.} \mathrm{Величина} e \varphi^{*}\left(E_{l}\right)(1)$ легко измеряется и в зависимости от диаметра контакта принимает следующие значения: $e \varphi_{1}^{*}(D=5$ мкм $)=5.14$ эВ, $e \varphi_{2}^{*}(D=10$ мкм $)=5.18$ эВ, $e \varphi_{3}^{*}(D=40$ мкм $)=5.21$ эВ и $е \varphi_{4}^{*}(D=120$ мкм $)=5.34$ эВ.

Изучение электростатической системы TiAlNiAu/ $n^{+}$-GaN омических контактов (рис. $3, a$ ) показало, что их поверхностный потенциал практически не отличается от электростатического потенциала свободной полупроводниковой поверхности $n^{+}-\mathrm{GaN}$ (рис. $3, b$ ), а электростатическое поле в области контакта и по его периферии практически равно нулю (рис. $3, d$ ). Это указывает на то, что TiAlNiAu-металлизация омического контакта находится в термодинамическом равновесии практически со всем объемом контактного слоя $n^{+}-\mathrm{GaN}$ и, как следствие, на отсутствие в нем барьера Шоттки. Согласно общепринятым представлениям, прохождение электрического тока в таких (без барьера или с очень низким барьером) ОК реализуется по термоэмиссионному механизму на основе диффузионного переноса подвижных носителей электрических зарядов [31]. 

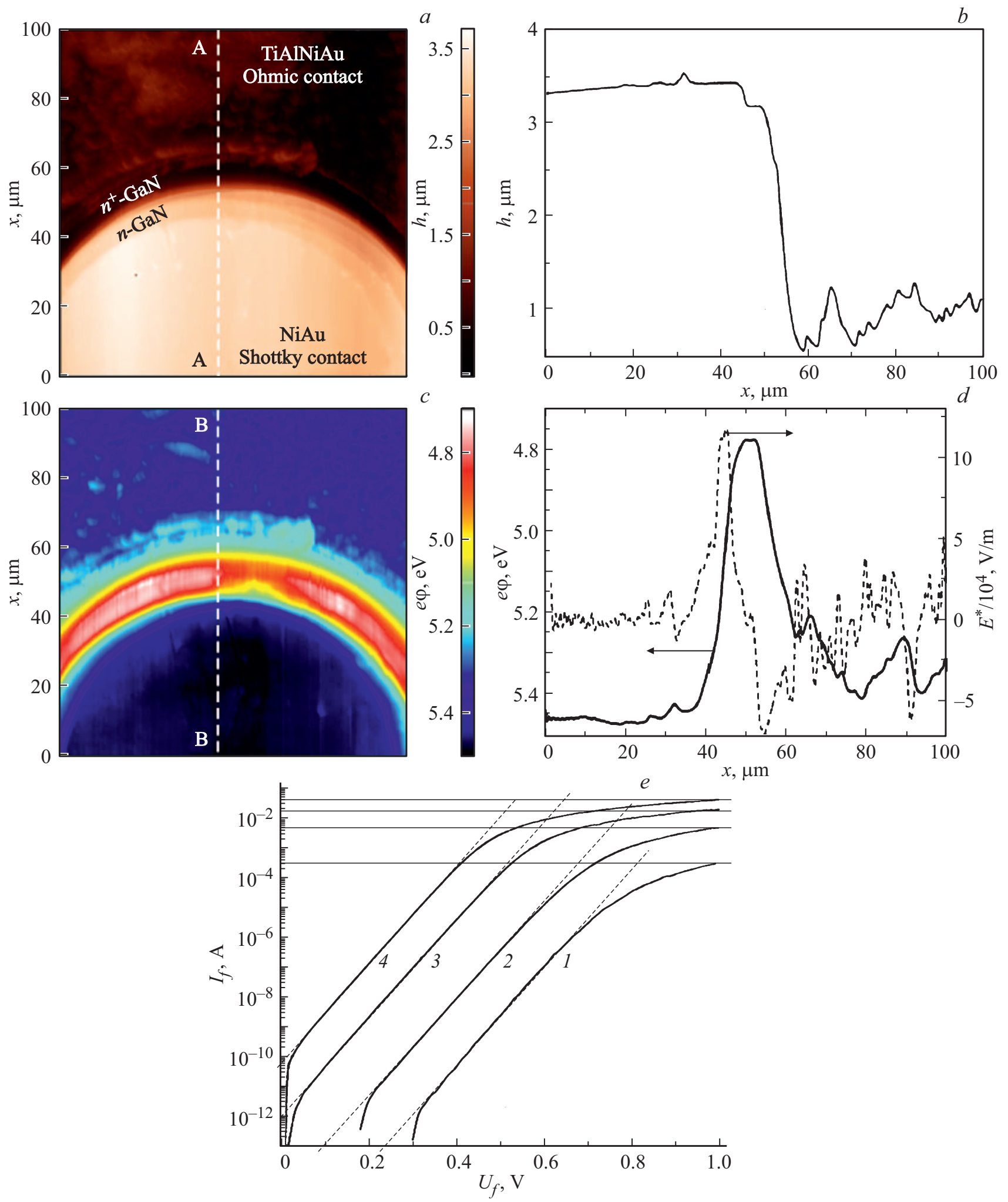

Рис. 1. АCM-изображения $\mathrm{AuNi} / n-n^{-}$-GaN-контакта Шоттки $D=120$ мкм и окружающего его TiAlNiAu/nGaN омического контакта: рельефа $h(x, y)(a)$ и профиля $h(x)$ его поперечного сечения $\mathrm{A}-\mathrm{A}(b)$, латерального распределения $е \varphi(x, y)(c)$ и профиля $е \varphi(x)$ его поперечного сечения $\mathrm{B}-\mathrm{B}(d) . c$ - прямые $\mathrm{BAX}$ диодных $\mathrm{AuNi} / n-n^{+}-\mathrm{GaN}$ контактов Шоттки, $D$, мкм: $1-5$, $2-10,3-40$ и $4-120$. 

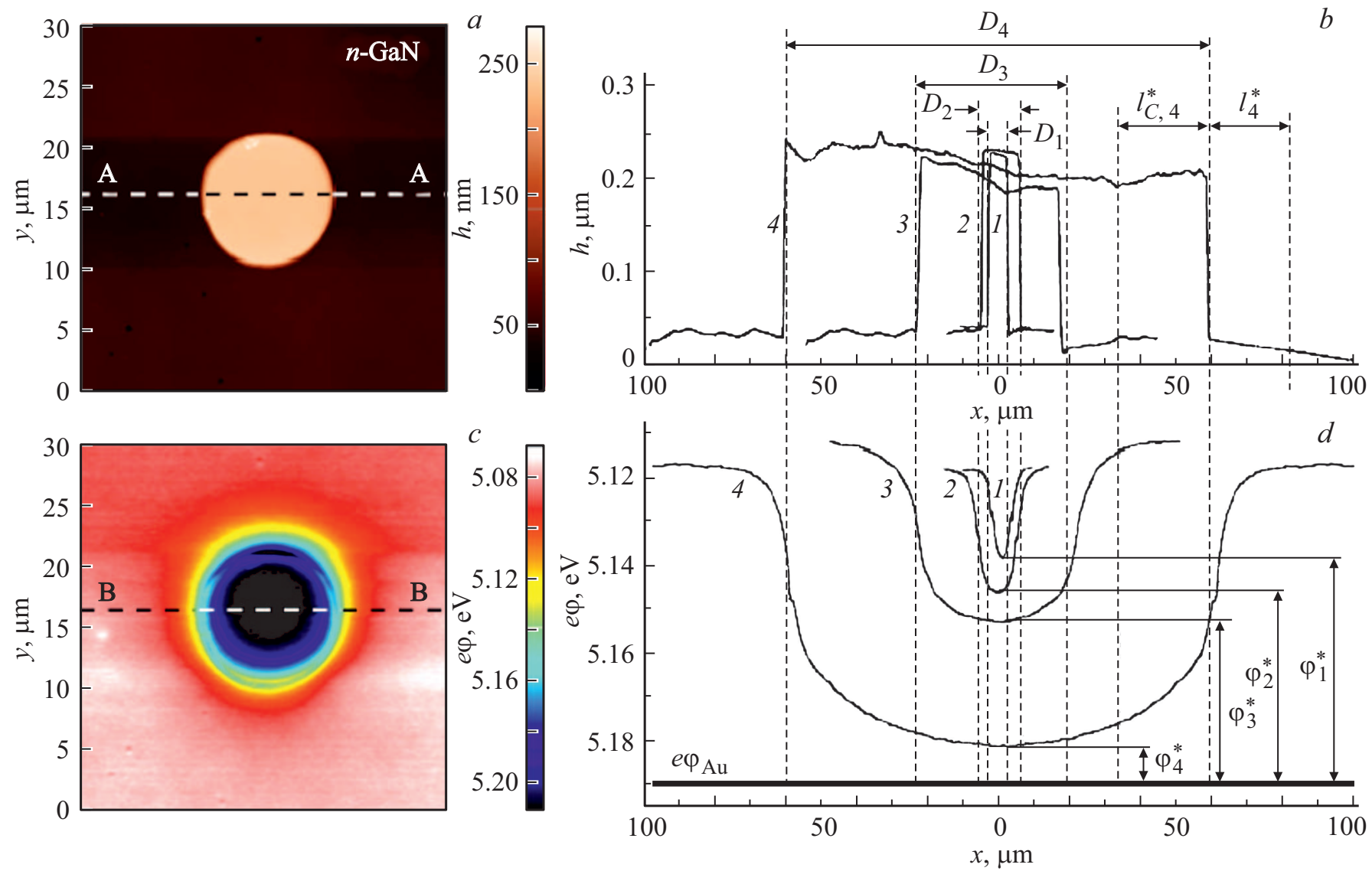

$x, \mu \mathrm{m}$

Рис. 2. АСМ-изображения рельефа $h(x, y) \mathrm{AuNi} / n-\mathrm{GaN}$-контакта Шоттки $D=10$ мкм $(a)$ и профилей $h(x)$ поперечных сечений (А-А) аналогичных контактов Шоттки, $D$, мкм: $1-5,2-10,3-40$ и $4-120$ (b). АСМ-изображение латерального распределения $е \varphi(x, y)$ контакта Шоттки $D=10$ мкм $(c)$ и профилей $е \varphi(x)$ поперечных сечений $(\mathrm{B}-\mathrm{B})$ аналогичных контактов Шоттки, $D$, мкм: $1-5,2-10,3-40$ и $4-120(d)$.

Данный факт находится в противоречии с известными утверждениями о том, что перенос электронов в омических контактах TiAlNiAu $/ n^{+}-\mathrm{GaN}$ происходит преимущественно по полевому механизму сквозь туннельнопрозрачный барьер Шоттки с пониженной эффективной высотой барьера $\varphi_{b, \text { eff }}$ [32]. Для окончательного ответа на вопрос о механизмах токопрохождения в омических контактах TiAlNiAu $/ n^{+}-\mathrm{GaN}$, по всей вероятности, необходимо проведение более подробных исследований.

\section{4. Обсуждение результатов}

Из [17] следует, что форма плоских контактов Шоттки играет важную роль в определении их основных приборных характеристик: токов насыщения $I_{S}$, емкостей $C$, высот потенциальных барьеров - $\varphi_{b}$, последовательных сопротивлений $R_{S}$, токовой $\beta_{I}$ и вольтовой $\beta_{U}$ фоточувствительностей и др. Напомним, что плоский контакт Шоттки бесконечной площади $S \rightarrow \infty$ не имеет выпрямляющих характеристик. Значение его $R_{S}$ стремится к нулю, а $C$ к бесконечности. Приборные характеристики у плоских контактов $\mathrm{M}-П$ появляются только после придания им определенной формы с периметром $P$ и площадью $S$.

На возможность влияния периферии плоских контактов М-П с БШ на их электростатическую систему впервые было обращено внимание в монографии Мамедова [4]. В частности, там указывалось на то, что вокруг периметра металлических контактов Шоттки существует дополнительное электростатическое поле $E_{D}$, образованное разностью поверхностных потенциалов металлического контакта $\varphi_{m}$ и свободной полупроводниковой поверхности $\varphi_{S}$. Согласно [4], вектор напряженности $\mathbf{E}_{D}$ направлен противоположно вектору напряженности электростатического поля ОПЗ $\mathbf{E}_{W}$. Максимальное значение модуля $|\mathbf{E} D|=\mathbf{E}_{D}=$ const постоянно, равно $\left|\mathbf{E}_{W}\right|$, определяется только природой контакта и никак не связано с его формой - ни с их площадью $S$, ни с периметром $P$.

Согласно [17], наличие только $\mathbf{E}_{D} \equiv \mathbf{E}_{\text {grad }}$ недостаточно для объяснения зависимости поверхностного потенциала плоского контакта Шоттки от его линейных размеров [17]. Там же сообщалось, что вокруг периметра контактов М-П с БШ, кроме постоянного $\left|\mathbf{E}_{\text {grad }}\right|=\left|\mathbf{E}_{W}\right|=$ const, существует еще выходящее в по- 

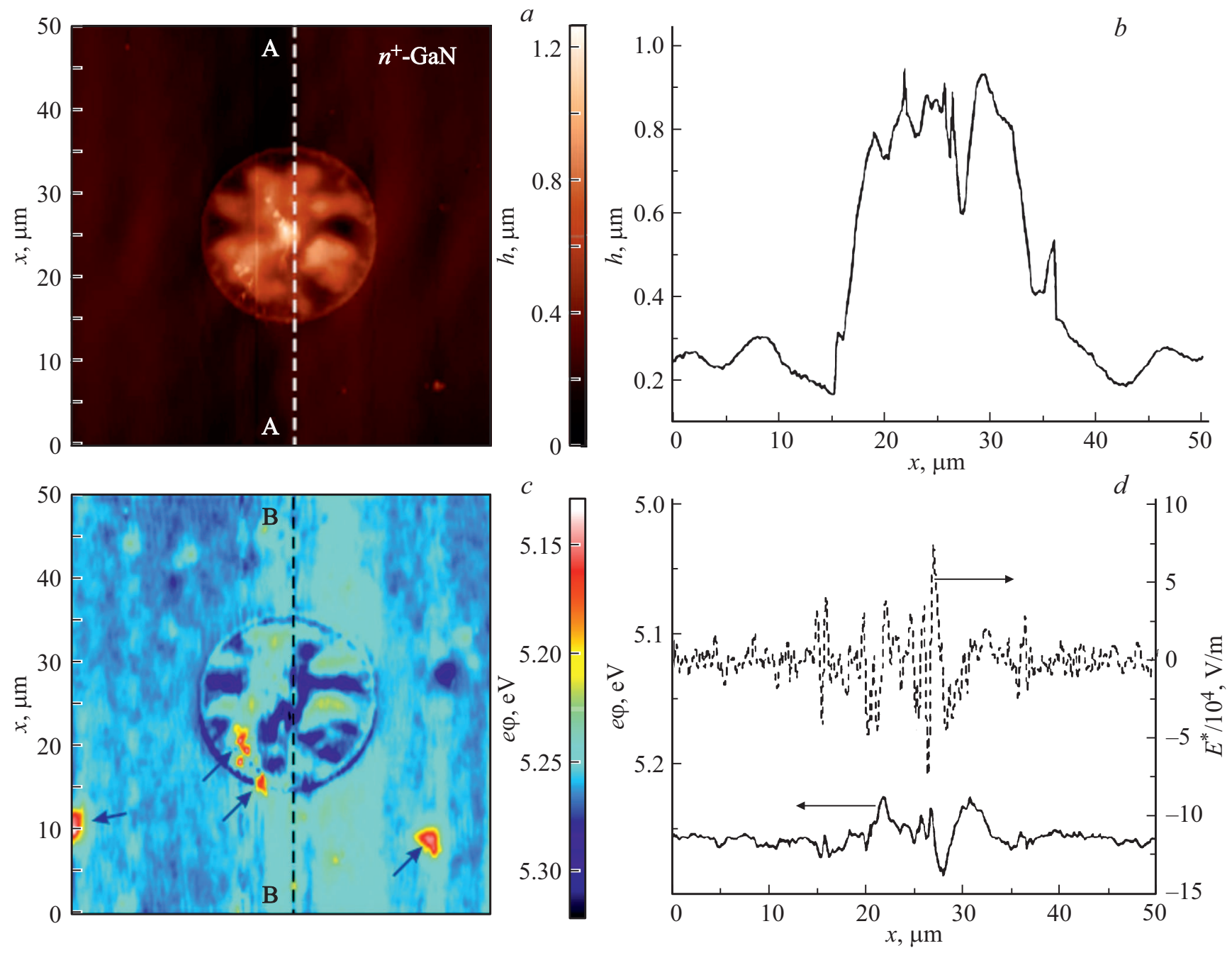

Рис. 3. АСМ-изображение поверхности tiAlNiAu $/ n^{+}-\mathrm{GaN}$ омического контакта $D=15$ мкм: рельефа поверхности $h(x, y)(a)$ и профиля $h(x)$ его попереченого сечения $(\mathrm{A}-\mathrm{A})(b)$, латерального распределения $е \varphi(x, y)$ (стрелками указан электростатический потенциал адсорбата) $(c)$ и профиля $е \varphi(x)$ его поперечного сечения $(\mathrm{B}-\mathrm{B})(d)$.

лупроводник через боковую поверхность ОПЗ и зависящее от площади и периметра контакта нескомпенсированное электростатическое поле $\mathbf{E}^{*}$ (рис. 1, $d$ и 4). В данном случае вектор $\mathbf{E}_{\text {grad }}$ сонаправлен с вектором электростатического поля $\mathbf{E}_{W}$ (рис. $4, a, \mathrm{SCh}$ ). В общем случае, для заданной системы М-П максимальное значение $\left|\mathbf{E}_{\text {grad }}\right|=\left|\mathbf{E}_{W}\right|$, а среднее значение $\left\langle\left|\mathbf{E}_{\text {grad }}\right|\right\rangle$ :

$$
\left\langle\left|\mathbf{E}_{\text {grad }}(\mathbf{r})\right|\right\rangle=\frac{\varphi_{\mathrm{Au}, \text { film }}-\varphi_{S}(x)}{\Delta \mathbf{r}} .
$$

Вектор $\mathbf{E}^{*}$, согласно теореме Остроградского-Гаусса, определяется плотностью потока вектора смещения электростатического поля через боковую поверхность ОП3 [30]. Площадь боковой поверхности ОПЗ можно рассчитать как $S^{*}=W_{\mathrm{SCh}} \cdot P$, где $W_{\mathrm{SCh}}-$ ширина ОПЗ при нулевом смещении.

Для плоских круглых контактов Шоттки уменышение диаметра $D$ приводит к квадратичному уменьшению площади $S \sim D^{2}$ и, соответственно, полного электрического заряда $Q_{W}$ ОПЗ. Площадь же боковой поверхности ОПЗ при этом уменьшается линейно, как $S^{*}=W_{\mathrm{SCh}} \cdot \pi D / 2$. Согласно теореме Остроградского-Гаусса, это ведет к уменьшению плотности потока вектора смещения электростатического поля через боковую поверхность ОП3, уменьшению модуля $\left|\mathbf{E}^{*}\right|$ и, согласно (2), увеличению $\left|\mathbf{E}_{l}\right|$, что приводит к увеличению встроенного в контакт электростатического потенциала $\varphi^{*}$ и уменьшению работы выхода электронов $A_{e}$ с Au-поверхности AuNi-контактов Шоттки.

Для плоских контактов больших площадей $S \rightarrow \infty$ модуль $\left|\mathbf{E}^{*}\right| \approx\left|\mathbf{E}_{\text {grad }}\right|$ максимален, а, согласно (2), $\left|\mathbf{E}_{l}\right| \rightarrow 0$ минимально и, соответственно, $\varphi^{0} \rightarrow 0$ имеет минимальное значение. Поэтому, как показывают эксперименты, для $\mathrm{AuNi} / n-\mathrm{GaN}$-контактов Шоттки с большими диаметрами $D \geq 120$ мкм поверхностный потенциал плоских металлических контактов (работа выхода $A_{e}$ ) практически не зависит от их размеров и приближается к работе выхода сплошной металлической пленки. 
Для понимания вышеизложенного рассмотрим термодинамическую систему выпрямляющих контактов М-П с БШ.

Необходимым условием приведения неравновесных систем в состояние термодинамического равновесия является наличие между ними механизмов обмена неравновесными частицами.

Такие механизмы между металлическим контактом и полупроводником возникают при достаточном их сближении. В результате этого контактирующие области металлического контакта и полупроводника за время релаксации $\tau$ обмениваются подвижными неравновесными носителями электрических зарядов (электронами и дырками) и приходят в состояние термодинамического равновесия, формируя таким образом равновесные области пространственного заряда в полупроводнике шириной $W_{\mathrm{SCh}}$ и слоя Томаса-Ферми в металлическом контакте $W_{\mathrm{TF}}$ (рис. 4,a). Данный процесс сопровождается выравниванием уровней Ферми $E_{\mathrm{F}, \mathrm{SCh}}=E_{\mathrm{F}, \mathrm{TF}}$ на интерфейсе контакта и формированием вокруг него экранирующего электростатического поля $E_{W}$, препятствующего дальнейшему переносу подвижных частиц в неравновесные области и переходу остальных объемов контактирующих металла и полупроводника в состояние термодинамического равновесия.

Релаксация системы металл-полупроводник исследуемых контактов $\tau-$ время перехода в состояние термодинамического равновесия составляет $\tau=1 /\left(2 \omega f_{\text {th }}\right)$ [33]. С учетом измеренных емкостей $C$ и последовательных сопротивлений $R_{s}$ исследуемых контактов их предельные частоты $f_{\text {th }}$ на 5-6 порядков больше резонансной частоты колебаний балки кантилевера $f_{\text {res }}=400$ кГц. Это означает, что менее чем за одну миллионную периода колебания балки кантилевера система контакта металл-полупроводник с БШ за счет протекания токов насыщения $I_{S}=10^{-10}-10^{-16} \mathrm{~A}$ (рис. $1, e$ ) успеет прийти в состояние термодинамического равновесия и выравнивания уровней Ферми $E_{\mathrm{F}, \mathrm{SCh}}=E_{\mathrm{F}, \mathrm{TF}} \quad$ на интерфейсе. Согласно [34], в этом случае становится возможным измерение с достаточной точностью компенсирующего напряжения $U_{0}=\Delta \varphi=\varphi_{\text {Au-Contact }}-\varphi_{\text {Au-Cantileve }}$ в измерительной схеме ACM, равного по значению КРП между поверхностными потенциалами Аu-контакта и острием иглы кантилевера. В отсутствие токов компенсирующее напряжение $U_{0}$ будет полностью соответствовать контактной разности поверхностных потенциалов без дополнительного падения напряжения на ОПЗ и приповерхностной области. Падением напряжения на ОПЗ, например, нельзя пренебречь в тех случаях, когда резонансная частота колебаний балки кантилевера соизмерима с частотой релаксации измеряемой системы при протекании выравнивающих токов, или в случае отсутствия токов насыщения $I_{S}$. Последнее противоречит теории выпрямляющих контактов и практическим результатам.

Те области полупроводника и металлического контакта, между которыми не произошел обмен электро-

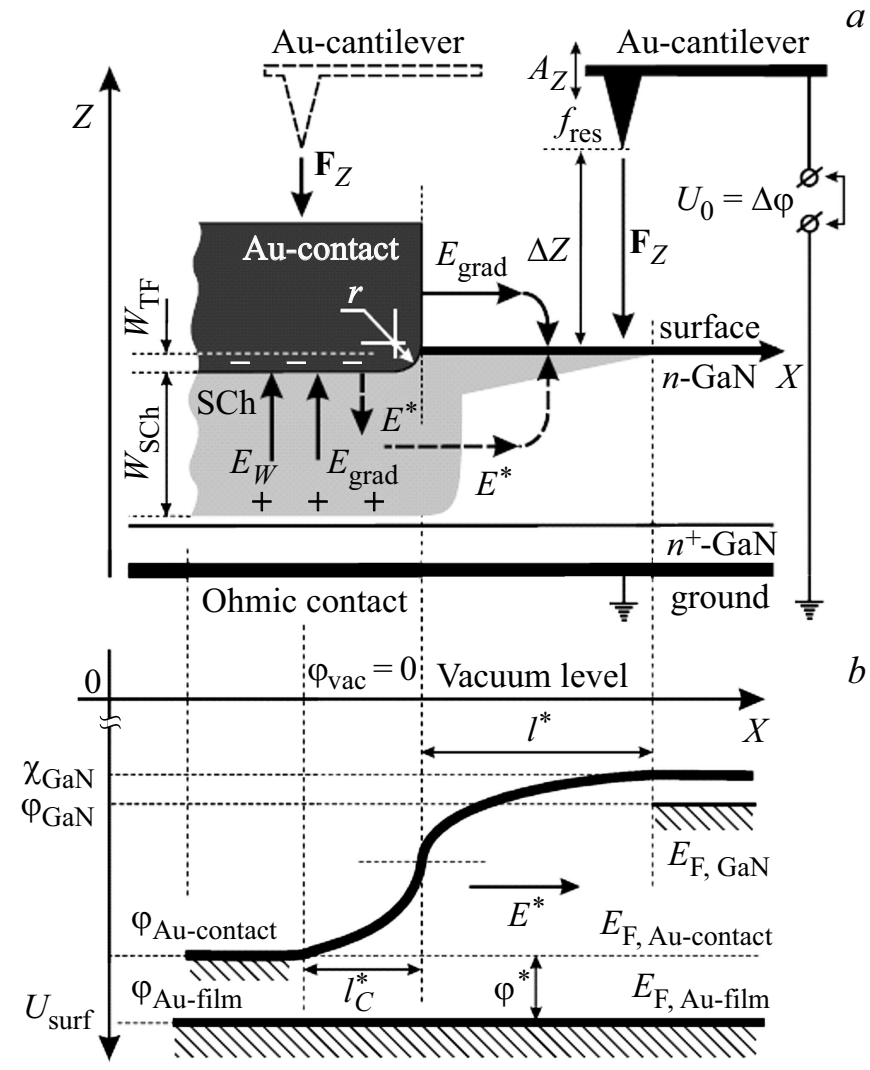

Рис. 4. Схематическое изображение способа измерения методом зонда Кельвина АСМ электростатического потенциала поверхности контакта металл-полупроводник с барьером Шоттки $(a)$ и латеральное распределение его поверхностного потенциала $\varphi(x)(b)$.

нами по причине возникновения в ОПЗ экранирующего поля $E_{W}$, являются неравновесными по отношению друг к другу и, соответственно, будут иметь разный поверхностный электростатический потенциал и работу выхода, что мы и наблюдаем в экспериментах.

Согласно вышеизложеному, внешнее нескомпенсированное электростатическое поле $\mathbf{E}^{*}$ будет также участвовать в перераспределении частиц и внутренней энергии в окружающем AuNi-контакт тонком приповерхностном $n$-слое полупроводника в данном случае $n$-GaN, что приводит к изменению его поверхностного электростатического потенциала в окружающем контакт ореоле $l^{*}$. Значение $\mathbf{E}^{*}$ может быть непосредственно измерено АСМ-методом Кельвина. Такое измерение возможно потому, что $\mathbf{E}^{*}$ входит в состав электрического контура измерительной системы атомно-силового микроскопа: земля-ОП3-поверхность- $\mathrm{F}_{Z}-$ кантилеверземля (graund $-\mathrm{SCh}-$ surface $-\mathrm{F}_{Z}-$ cantilever - graund) и, согласно условиям термодинамического равновесия, принимает участие в механизмах обмена заряженными частицами измеряемого участка поверхности с иглой кантилевера через заземленный graund-контакт (рис. 4, $a$ ). 
Кроме внешнего ореола $l^{*}$, на рис. $2, d$ показано наличие другой переходной области внутри периметра $\mathrm{AuNi- \kappa онтакта} \mathrm{-} \mathrm{внутренний} \mathrm{ореол} \mathrm{протяженностью} l_{C}^{*}$. Согласно рис. $2, d$, внутренний ореол $l_{C}^{*}$ определяет эффективную периферийную область контакта, в которой влияние $\mathbf{E}_{l}$ на контакт максимально [17].

Электростатический потенциал, формируемый другой составляющей электростатического поля периферии $E_{\text {grad, }}$ в АСМ-методе зонда Кельвина напрямую измерить невозможно по причине того, что поле $E_{\text {grad }}$, согласно рис. $4, b$, возникает в другом замкнутом электрическом контуре контакт-поверхность-ОПЗ-контакт (contact-surface-SCh-contact), не входящем в состав измерительной электрической схемы АСМ. О наличии $E_{\mathrm{grad}}$ в системе контакта металл-полупроводник можно судить лишь исходя из косвенных результатов измерений значений электростатических потенциалов поверхностей металлических контактов $\varphi_{\text {contact }}$ и полупроводниковой поверхности $\varphi_{\mathrm{GaN}}$.

\section{5. Заключение}

Предлагаемая физическая модель электростатической системы плоских контактов М-П с БШ на широкозонных полупроводниках основана на значительном влиянии периферийной области контакта на формирование его основных электрофизических и приборных характеристик. Столь сильное влияние периферии осуществляется посредством электростатического поля периферии $\mathbf{E}_{l}$, образованного суперпозицией выходящего за боковую поверхность ОПЗ и зависящего от линейных размеров контакта нескомпенсированного электростатического поля $\mathbf{E}^{*}$ и электростатического поля, образованного градиентом между поверхностными потенциалами металлического контакта и свободной полупроводниковой поверхностью $\mathbf{E}_{\text {grad. }}$. Для заданной системы контакта M-П с БШ $\left|\mathbf{E}_{\text {grad }}\right|=$ const - постоянно (определяется только физическими свойствами системы М-П), а $\left|\mathbf{E}^{*}\right|$ изменяется в зависимости от формы и размеров контакта [17].

Данная модель может использоваться в качестве дополнения к физическим моделям плоских выпрямляющих контактов металл-полупроводник ШотткиМотта [35], или Бардина [36].

Обнаруженные принципиальные отличия термодинамических и электростатических систем омических TiAlNiAu $/ n^{+}-\mathrm{GaN}$-контактов (в отличие от аналогичных систем $\mathrm{AuNi} / n-\mathrm{GaN}$-контактов Шоттки) указывают на отсутствие в них барьера Шоттки и преобладающую роль термоэмиссионного механизма переноса подвижных носителей электрических зарядов.

Из вышеизложенного следует, что наибольшее влияние встроенные электростатические поля периферии будут оказывать на электростатическую систему выпрямляющих контактов М-П микронных и наноразмеров.

\section{Финансирование работы}

Работа выполнена при финансовой поддержке ПНИЭР „Исследование и разработка технологии изготовления сверхвысокочастотных монолитных интегральных схем на основе гетероструктур InAlN/GaN для изделий космического применения“" № 14.578.21.0240 от 26.09.2017 г. УИР REMЕFI 57817Х240.

\section{Конфликт интересов}

Авторы заявляют, что у них нет конфликта интересов.

\section{Список литературы}

[1] S.M. Sze, Kwok K. Ng. Physics of Semiconductor Devices, 3nd edn (John Wiley\& Sons, Inc., Publication, 2007) p. 764.

[2] R.T. Tung. Appl. Phys. Rev., 1, 011304 (2014).

[3] R.T. Tung. Mater. Sci. Engin. R: Reports, 35, 1 (2001).

[4] Р. Мамедов. Контакты металл-полупроводник с электрическим полем пятен (Баку, БГУ, 2003).

[5] В.Г. Божков. Контакты металл-полупроводник: физика и модели (Томск, Изд. Дом Томского гос. ун-та, 2016).

[6] Н.А. Торхов, С.В. Еремеев. ФТП, 34 (1), 106 (2000).

[7] Н.А. Торхов. ФТП, 35 (7), 823 (2001).

[8] Л.А. Косяченко, А.В. Макаров, С.Э. Остапов, И.М. Раренко. Технология и конструирование в электронной аппаратуре, 3, 3 (2002).

[9] Rüdiger Quay. Gallium Nitride Electronics (Springer Series in Materials Sciences. 2008) p. 492. DOI: 10.1007/978-3-54071892-5.

[10] Nitride Semiconductor Devices. Principles and Simulation, ed. by Joachim Piprek (Wiley-VCH Verlag GmbH\& Co. KGaA, 2007).

[11] M.D. Barlow. Metal-Semiconductor Contacts for Schottky Diode Fabrication (Youngstown State University, 2007).

[12] M. Siva Pratap Reddy, Hee-Sung Kang, Jung-Hee Lee, V. Rajagopal Reddy, Ja-Soon Jang. J. Appl. Polym. Sci., 131 39773 (2014). DOI: 10.1002/app.39773

[13] M.G. Helander, Z.B. Wang, J. Qiu, Z.H. Lu. Appl. Phys. Lett., 93, 193310 (2008).

[14] Moh'd Rezeq, Khouloud Eledlebi, Mohammed Ismail, Ripon Kumar Dey, Bo Cui. J. Appl. Phys., 120, 044302 (2016).

[15] R.H. Fowler. Phys. Rev., 38, 45 (1931).

[16] H. Elabd, W. Kosonoky. RCA Rev., 43, 569 (1982).

[17] Н.А. Торхов. ФТП, 52 (10), 1150 (2018).

[18] K.L. Sorokina, A.L. Tolstikhina. Crystallography Reports, 49 (3), 476 (2004).

[19] M. Wicinski, W. Burgstaller, A.W. Hassel. Corrosion Sci., 104, 1 (2016).

[20] S.Y. Luchkin, K.J. Stevenson. Microscopy and Microanalysis, 24 (2), 126 (2018). DOI: 10.1017/S1431927618000156

[21] Н.М. Коровкина. Автореф. канд. дис. (СПб., РГБб, 2006).

[22] Н.А. Торхов, В.А. Новиков. ФТП, 45 (1), 69 (2011).

[23] Н.А. Торхов. ФТП, 44 (5), 1 (2010).

[24] Н.А. Торхов, В.Г. Божков, И.В. Ивонин, В.А. Новиков. Поверхность, 11, 1 (2009)ю

[25] Н.А. Торхов, В.Г. Божков, С.М. Гущин, В.А. Новиков. В сб.: Тез. докл. 22-я Междунар. Крымская конф. „СВЧ-техника и телекоммуникационные технологии“ (Крым, Украина, 2012) c. 635 . 
[26] Н.А. Торхов, В.Г. Божков, В.А. Новиков, А.А. Мармалюк, Ю.Л. Рябоштан. В сб.: Тез. докл. 22-я Междунар. Крымская конф. „СВЧ-техника и телекоммуникационные технологии“ (Крым, Украина, 2012) с. 633.

[27] Н.А. Торхов, В.Г. Божков, В.А. Новиков, А.А. Мармалюк, Ю.Л. Рябоштан. В сб.: Тез. докл. 25-я Междунар. Крымская конф. „СВЧ-техника и телекоммуникационные технологии“ (Крым, Россия, 2015) с. 611.

[28] Н.А. Торхов, В.А. Новиков, В.Н. Брудный. В сб.: Тез. докл. VII Всеросс. науч.-техн. конф. „Обмен опытом в области создания сверхширокополосных радиоэлектронных систем“ (Омск, 2018) с. 296.

[29] Н.А. Торхов. ФТП, 45 (7) 965 (2011).

[30] Н.А. Торхов. Изв. вузов. Физика. Деп. в ВИНИТИ. № 334B2008 от 18.04.2008.

[31] Т.В. Бланк, Ю.А. Гольдберг. ФТП, 41 (11), 1281 (2007).

[32] S.N. Mohammad. J. Appl. Phys., 95 (12), 7940 (2004).

[33] N.A. Torkhov, L.I. Babak, A.A. Kokolov, A.S. Salnikov, I.M. Dobash, V.A. Novikov, I.V. Ivonin. J. App. Phys., 119, 094505 (2016).

[34] В.Л. Миронов. Основы сканирующей зондовой микроскопии (Техносфера, Н. Новгород, 2004) с. 144.

[35] W.E. Meyerhof. Phys. Rev., 71 (10), 727 (1947).

[36] E.H. Rhoderick, R.H. Williams. Metall-semiconductor contacts, 2nd ed. (Clarendon, Oxford, 1988).

Редактор Г.А. Оганесян

\title{
Study of the electrostatic system of the surface of AuNi/GaN Schottky diodes by the Kelvin Probe Force Microscopy
}

\author{
N.A. Torkhov ${ }^{1,2,3}$, V.A. Novikov ${ }^{2}$ \\ ${ }^{1}$ Scientific-Research Institute of Semiconductors, \\ 634034 Tomsk, Russia \\ 2 Tomsk State University, \\ 634050 Tomsk,Russia \\ 3 Tomsk State University \\ of Control System and Radioelecrtronics, \\ 634050 Tomsk, Russia
}

Abstract The AFM investigation of electrostatic system of crystal surface of flat $\mathrm{AuNi} / n-n^{+}-\mathrm{GaN}$ Schottky diode shown that the value of work function for the electron dependence on the lateral dimention (diameter $-D$ ) of metal Schottky contacts. For $D>120 \mu \mathrm{m}$ value of work function of the electron in the center of contact almost equal to value of work function of infinite metal film $-e \varphi_{\mathrm{Au}} \approx 5.40 \mathrm{eV}$. If diameter of contact decreases then value of work function of the electron in the center of contact decreases also: $5.34 \mathrm{eV}$ for $D=120 \mu \mathrm{m}, 5.21 \mathrm{eV}$ for $D=40 \mu \mathrm{m}, 5.18 \mathrm{eV}$ for $D=10 \mu \mathrm{m}$ and $5.14 \mathrm{eV}$ for $D=5 \mu \mathrm{m}$. This effect is associated with influence inbuilt electric field of periferia $b f E_{l}$, which depend on area $S$ and perimeter $P$ of Schottky contacts. The investogation of electrostatic system of ohmic contacts TiAlNiAu $/ n^{+}-\mathrm{GaN}$ by Kelvin probe mycroscopy shown that for carriers of charge the dominant role is a diffusion mechanism. 